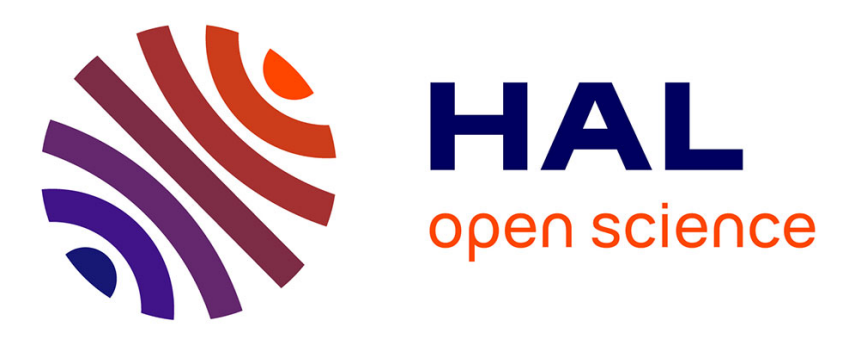

\title{
Approximating the vanishing capillarity limit of two-phase flow in multi-dimensional heterogeneous porous medium
}

\author{
Boris Andreianov, Konstantin Brenner, Clément Cancès
}

\section{To cite this version:}

Boris Andreianov, Konstantin Brenner, Clément Cancès. Approximating the vanishing capillarity limit of two-phase flow in multi-dimensional heterogeneous porous medium. Journal of Applied Mathematics and Mechanics, 2014, 94 (7-8), pp.651-667. hal-00744359

\section{HAL Id: hal-00744359 \\ https://hal.science/hal-00744359}

Submitted on 23 Oct 2012

HAL is a multi-disciplinary open access archive for the deposit and dissemination of scientific research documents, whether they are published or not. The documents may come from teaching and research institutions in France or abroad, or from public or private research centers.
L'archive ouverte pluridisciplinaire HAL, est destinée au dépôt et à la diffusion de documents scientifiques de niveau recherche, publiés ou non, émanant des établissements d'enseignement et de recherche français ou étrangers, des laboratoires publics ou privés. 


\title{
Approximating the vanishing capillarity limit of two-phase flow in multi-dimensional heterogeneous porous medium
}

\author{
Boris Andreianov* Konstantin Brenner ${ }^{\dagger} \quad$ Clément Cancès $^{\ddagger}$
}

\begin{abstract}
Neglecting capillary pressure effects in two-phase flow models for porous media may lead to non-physical solutions: indeed, the physical solution is obtained as limit of the parabolic model with small but non-zero capillarity. In this paper, we propose and compare several numerical strategies designed specifically for approximating physically relevant solutions of the hyperbolic model with neglected capillarity, in the multi-dimensional case.

It has been shown in [Andreianov \& Cancès, Comput. Geosci., 2013, to appear] that in the case of the one-dimensional Buckley-Leverett equation with distinct capillary pressure properties of adjacent rocks, the interface may impose an upper bound on the transmitted flux. This transmission condition may reflect the oil trapping phenomenon. We recall the theoretical results for the one-dimensional case which are used to motivate the construction of multidimensional finite volume schemes. We describe and compare a coupled scheme resulting as the limit of the scheme constructed in [Brenner \& Cancès \& Hilhorst, HAL preprint no.00675681, 2012) and two IMplicit Pressure - Explicit Saturation (IMPES) schemes with different level of coupling.
\end{abstract}

Keywords two-phase flow, heterogeneous porous medium, discontinuous capillarity, vanishing capillarity, Buckley-Leverett model, oil trapping, admissible solution, finite volume approximation

MSC2010 subject classification $76 \mathrm{~S} 05,35 \mathrm{~L} 65,65 \mathrm{M} 08$

\section{Introduction}

Consider a flow in porous medium of a mixture of two immiscible phases (typically, oil and water). In this note, we start with a well-established parabolic-elliptic model that accounts for capillarity forces through a nonlinear function $\pi(x, s)$ where $x$ is a point location (the dependence on $x$ accounts for physical properties of the medium) and $s$ is the saturation of one of the phases. In this case, the flow is driven by convection (first-order) and diffusion/capillarity (second order) forces. Yet in large time and space scales which typically occur in geological applications, it is tempting to

\footnotetext{
*Laboratoire de Mathématiques CNRS UMR 6623, Université de Franche-Comté, 16 route de Gray 25030 Besançon Cedex, France. boris.andreianov@univ-fcomte.fr

${ }_{\dagger}^{\dagger}$ Laboratoire Jean-Alexandre Dieudonné, Université de Nice Sophia Antipolis \& team Coffee INRIA Sophia Antipolis Méditerranée, 06108 Nice Cedex 02, France. konstantin.brenner@unice.fr

¥UPMC Univ Paris 06 \& CNRS, UMR 7598, Laboratoire Jacques-Louis Lions, Boite courrier 187, 4 place Jussieu, 75252 Paris Cedex 05, France, cances@ann.jussieu.fr
} 
neglect the second-order effects. Formally, this results in a hyperbolic-elliptic system of equations. In one space dimension the elliptic equation is integrated explicitly, and the resulting BuckleyLeverett equation is a hyperbolic scalar conservation law. In the case of heterogeneous medium with sharp transitions between layers, both theory and numerical approximation of this equation were deeply investigated in recent years. In the present paper, our goal is to benefit both from the understanding of the coupling conditions at the interface between multidimensional porous media [17] and the understanding of the relevant hyperbolic limit in the one-dimensional case [4] in order to propose a consistent numerical strategy for the hyperbolic-elliptic model that appears in dimensions two and three. Starting from the the scheme exhibited in the work [8], we build and test numerical schemes that agree with the vanishing capillarity paradigm.

Let us first discuss the one-dimensional case following the references $[4,13,14,24,25]$. In these works, the Buckley-Leverett equation is seen as the singular limit of parabolic equation with capillarity or viscosity terms. The resulting solutions are characterized intrinsically as entropy solutions. In general, entropy solutions are weak (distributional) solutions satisfying additional integral inequalities that single out the physically relevant solution. In particular, in the fundamental case of a homogeneous medium, and also in the case of a heterogeneous medium with gradually changing physical properties, the classical Kruzhkov theory of entropy solutions [25] fully describes the vanishing capillarity limit (see, e.g., [24] and references therein).

The situation is very different when a jump heterogeneity is present in the medium, as this is the case at the interface between two different geological layers. In this case, we deal with a discontinuous-flux Buckley-Leverett equation (see [1,4,10,24] and references therein) that we see as the limit, as $\varepsilon \rightarrow 0$, of the parabolic model

$$
\phi(x) s_{t}+f(x ; s)_{x}=\varepsilon\left(\lambda(x ; s) \pi(x, s)_{x}\right)
$$

with piecewise constant in $x$ functions $f, \lambda$ and $\pi$; we refer to Section 1 for details. The analysis of [15] (see [13,14] for more details) shows that, according to the relation between the capillarity parameters on the two sides of the rock, two qualitatively different kinds of solution may appear. The optimal entropy conditions (see $[1,24]$ ) select the weak solution of the discontinuous-flux BuckleyLeverett equation that facilitates the migration of the phases across the interface between the two layers. The barrier entropy conditions of [14] select the weak solution of the same equation that minimizes the flux of the phases across the interface. Notice that this latter case can be seen as a rough mathematical model for the oil trapping phenomenon, responsible for the formation of natural oil reservoirs. Furthermore, it was shown in [4] that infinitely many intermediate notions of entropy solution (accounting for a more or less stringent limitation of the flux conductivity of the interface) may appear at the vanishing capillarity limit. More exactly, given the convective fluxes $f_{l, r}(s)$ on the left $(l)$ and on the right $(r)$ from the interface, the appropriate entropy inequalities depend on the shape of capillary pressure functions $\pi_{l, r}(s)$. As a consequence, one may state that

the physically relevant solution of the Buckley-Leverett equation takes into account the shape of (formally neglected) capillary pressure functions.

Roughly speaking, in the relevant solution the values of capillary pressure on each side from the interface should coincide or be "connected" by a suitable interface layer (see [4] for details).

Due to this observation, the numerical approximation of the two-phase flow in heterogeneous media, at geological scales, becomes a particularly delicate issue. On the one hand, discretization of the full parabolic problem (as the one of [8]) may be too expensive with respect to the precision 
required. On the other hand, whenever the saturation is taken as the primal variable straightforward discretization of the singular limit must fail, because the formal equation at the limit does not contain any information on the capillary pressure functions; it becomes impossible to distinguish between "optimal", "barrier" and the intermediate kinds of solutions to the problem. Thus, in order to capture the physically relevant solution while discretizing the Buckley-Leverett equation, we have to complement a standard approximation strategy (such as fully implicit or IMplicit Pressure - Explicit Saturation (IMPES) scheme with time-explicit three-point finite volume approximation of the hyperbolic equation) with a specific procedure which goal is to connect the values of capillary pressure.

Such a scheme was developed in [4] (see also [5]) in the one-dimensional case. Yet the multidimensional situation is different, because an elliptic equation for the pressure cannot be eliminated from the system. Therefore, we start with the multi-dimensional scheme of [8], set the capillarity parameter $\varepsilon$ equal to zero, and introduce a series of simplifications aimed at reducing the computational complexity. The resulting schemes are compared numerically. Notice that, due to the low regularity of solutions of the equation giving fluid velocity, it does not seem possible to extend the theoretical study from the one-dimensional case to the multi-dimensional one. Thus, numerical evidence is the key ingredient of validation of the scheme, along with the convergence proof valid in the one-dimensional case only.

The paper is organized as follows. In Section 1 we describe the models. In Section 2 the information inferred from analysis of the one-dimensional case is outlined. Section 3 is the core of the paper: different versions of the finite volume scheme are presented. Section 4 provides numerical results.

\section{Description of the models}

In this paper, we consider a new model that is directly obtained from the one studied in $[8,17]$; while we neglect the capillary diffusion within the rocks, we keep track of the singular effect linked to the capillary pressure discontinuity at the interface. In Section 1.1, we briefly present the case of non-zero capillary diffusion whose contribution away from inner interfaces is neglected in the model given in Section 1.2.

In the sequel we assume that the porous medium $\Omega \subset \mathbb{R}^{d}$ is made of two different rocks $\Omega_{1}, \Omega_{2}$ separated by an interface $\Gamma=\partial \Omega_{1} \cap \partial \Omega_{2}$. For the sake of simplicity, we assume that both $\Omega_{i}$ are polygonal subdomains, and we restrict our study to the case of a finite time horizon $T>0$.

\subsection{The parabolic model}

Our starting point is the model where immiscible incompressible two-phase flow in the homogeneous porous medium $\Omega_{i}$ is governed by the coupling of the degenerate parabolic equation on the saturation

$$
\phi_{i} \partial_{t} s+\nabla \cdot\left(\mathbf{u}_{\mathrm{t}} f_{i}(s)+\underline{\mathbf{K}}_{i} \lambda_{i}(u)\left(-\nabla \pi_{i}(u)+\rho \mathbf{g}\right)\right)=q_{o}(s),
$$

with the uniformly elliptic equation

$$
\nabla \cdot \mathbf{u}_{\mathrm{t}}=q_{o}(s)+q_{w}(s), \quad \mathbf{u}_{\mathrm{t}}=-\underline{\mathbf{K}}_{i}\left(M_{i}(s) \nabla P-\zeta_{i}(s) \mathbf{g}\right) .
$$

In equations (2) and (3), $\phi_{i} \in(0,1)$ denotes the porosity, $s \in L^{\infty}(\Omega \times(0, T) ;[0,1])$ is the saturation of the oil phase, $\mathbf{u}_{t}$ is the total speed, the fractional flow $f_{i}(s)$ is an increasing function such 
that $f_{i}(0)=0$ and $f_{i}(1)=1, \underline{\mathbf{K}}_{i}$ is the intrinsic permeability tensor of the rock $\Omega_{i}, \lambda_{i}$ is a nonnegative function satisfying $\lambda_{i}(0)=\lambda_{i}(1)=0$ and $\lambda_{i}(s)>0$ for $s \in(0,1)$, the capillary pressure function $\pi_{i}$ is increasing and belongs to $L^{1}(0,1), \rho$ denotes the difference between the density of oil and the density of water, while $\mathbf{g}$ is the gravity. The oil and water source terms $q_{o}, q_{w}$ satisfy $q_{o}(0) \geq 0, q_{w}(1) \geq 0$. The function $M_{i}(s)$ is such that $M_{i}(s) \geq \alpha>0$ for all $s \in[0,1]$, and $\zeta_{i}(s)$ is a smooth function. The function $P$ is the so called global pressure which is a kind of weighted mean of the phase pressure [19]. We stress here that since $P$ is not a physical pressure, it can be discontinuous at the rock discontinuities.

Let us now focus on the transmission conditions across $\Gamma$. Due to conservation of mass, one has

$$
\sum_{i=1,2} \mathbf{u}_{\mathrm{t}} \cdot \mathbf{n}_{i}=0, \quad \sum_{i=1,2}\left(\mathbf{u}_{\mathrm{t}} f_{i}(s)+\underline{\mathbf{K}}_{i} \lambda_{i}(u)\left(-\nabla \pi_{i}(u)+\rho \mathbf{g}\right)\right) \cdot \mathbf{n}_{i}=0 .
$$

It has been pointed out in [17] that it is natural to extend the notion of phase pressure by considering $\pi_{i}$ as multivalued graphs, in order to give sense to the transmission conditions across rock discontinuities. Therefore, the capillary pressure has also to be considered as multivalued, yielding the introduction of the maximal monotone graph $\tilde{\pi}_{i}$ defined by

$$
\tilde{\pi}_{i}(s)=\pi_{i}(s) \text { if } s \in(0,1), \quad \tilde{\pi}_{i}(0)=\left[-\infty, \pi_{i}(0)\right] \quad \text { and } \quad \tilde{\pi}_{i}(1)=\left[\pi_{i}(1),+\infty\right]
$$

Requiring the "continuity" of the extended phase pressure turns to requiring the existence of a measurable function $\pi: \Gamma \times(0, T) \rightarrow \overline{\mathbb{R}}$ such that

$$
\pi \in \tilde{\pi}_{1}\left(s_{1}\right) \cap \tilde{\pi}_{2}\left(s_{2}\right) \quad \text { and } \quad P_{1}-Z_{1}(\pi)=P_{2}-Z_{2}(\pi),
$$

where $Z_{i}(p)=\int_{0}^{p} f_{i} \circ \tilde{\pi}_{i}^{-1}(a) \mathrm{d} a$ and $s_{i}, P_{i}$ denote the traces of $s, P$ on $\Gamma \times(0, T)$ from the side of $\Omega_{i}$.

The system (2)-(5) is complemented by an initial condition on the saturation

$$
s(x, 0)=s_{0}(x) \in[0,1] \text { for almost all } x \in \Omega,
$$

and by boundary conditions. Here we consider the simplest choice, i.e. no-flux boundary conditions:

$$
\left(\mathbf{u}_{\mathrm{t}} f_{i}(s)+\underline{\mathbf{K}}_{i} \lambda_{i}(u)\left(-\nabla \pi_{i}(u)+\rho \mathbf{g}\right)\right) \cdot \mathbf{n}=\mathbf{u}_{\mathrm{t}} \cdot \mathbf{n}=0 \quad \text { on } \quad \partial \Omega \cap \partial \Omega_{i} \times(0, T) .
$$

We could deal with more general boundary conditions, but this is beyond the scope of this paper.

Let us point out that under suitable assumptions on the data, system (2)-(7) admits at least one solution [17], and it can be approximated by means of a fully implicit Finite Volume scheme of $[8]$.

\subsection{The singular limit as capillarity is neglected}

As explained for example in $[3,4,15]$, rock heterogeneities strongly influence the behavior of the flow, since a singular effect can be induced at the rock discontinuities. Therefore our purpose is to consider the capillarity only at the rock discontinuity, and to neglect it within the homogeneous domain where it plays a minor role. From a practical point of view, we slightly modify equation (2) to get

$$
\phi_{i} \partial_{t} s+\nabla \cdot\left(\mathbf{u}_{\mathrm{t}} f_{i}(s)+\lambda_{i}(u) \rho \underline{\mathbf{K}}_{i} \mathbf{g}\right)=q_{o}(s),
$$


and we modify the flux continuity relations at the interface to get

$$
\sum_{i=1,2} \mathbf{u}_{\mathrm{t}} \cdot \mathbf{n}_{i}=0, \quad \sum_{i=1,2}\left(\mathbf{u}_{\mathrm{t}} f_{i}(s)+\underline{\mathbf{K}}_{i} \lambda_{i}(u) \rho \mathbf{g}\right) \cdot \mathbf{n}_{i}=0 \quad \text { on } \Gamma \times(0, T) .
$$

Finally, the boundary condition (7) takes the form

$$
\left(\mathbf{u}_{\mathrm{t}} f_{i}(s)+\lambda_{i}(u) \rho \underline{\mathbf{K}}_{i} \mathbf{g}\right) \cdot \mathbf{n}=\mathbf{u}_{\mathrm{t}} \cdot \mathbf{n}=0 \quad \text { on } \partial \Omega \cap \times(0, T) .
$$

Notice that for a given total speed $\mathbf{u}_{\mathrm{t}}$, equation (8) is a scalar conservation law. As noticed in [9], the no-flux boundary condition (10) is satisfied in a strong sense thanks to the fact that $\mathbf{u}_{\mathrm{t}} \cdot \mathbf{n}=0$ and $\lambda_{i}(0)=\lambda_{i}(1)=0$ for the flux function $\mathbf{F}$ defined by $\mathbf{F}(x, t ; s)=\mathbf{u}_{\mathrm{t}}(x, t) f_{i}(s)+\lambda_{i}(s) \rho \underline{\mathbf{K}}_{i} \mathbf{g}$.

In our simplified model, we should keep the formal jump conditions (5) so that the contribution of the capillarity is taken into account at the interface between the different rocks where it plays a crucial role. Let us stress that due to an interface layer that may develop as $\varepsilon \rightarrow 0$, (5) may not hold for the solutions we construct. Yet, as shows the study of the one-dimensional case (Section 2), it is correct to prescribe (5) at the level of the numerical approximation; as the discretization step goes to zero, a numerical boundary layer may be observed. Except in Section 2, we do not investigate the precise meaning that should be given to (5). Our goal is to solve numerically the problem described by equations (3),(5),(6),(8)-(10).

\section{Entropy solutions of the one-dimensional Buckley-Leverett model}

In the one-dimensional case, equation (3) implies that $\mathbf{u}_{\mathrm{t}}$ does not depend on the space variable, so that the model reduces to a single scalar conservation law with discontinuous flux function for the sole unknown $s$ (see $[1,2,7,10]$ and references therein for examples and general theory of such problems). Here we give a summary of the relevant analysis carried out in [4], with emphasis on the numerical recipe of flux limitation and introduction of the pressure transmission parameter $\pi$.

In [4], the Buckley-Leverett equation in piecewise homogeneous two-rocks' medium with interface situated at $\{x=0\}$ is seen as the singular limit, as $\varepsilon \rightarrow 0$, of the problems the form (1) with

$$
f(x, \cdot)=\left\{\begin{array}{l}
f_{l}(\cdot), x<0 \\
f_{r}(\cdot), x>0
\end{array}, \lambda(x, \cdot)=\left\{\begin{array}{l}
\lambda_{l}(\cdot), x<0 \\
\lambda_{r}(\cdot), x>0
\end{array}, \pi(x, \cdot)=\left\{\begin{array}{l}
\pi_{l}(\cdot), x<0 \\
\pi_{r}(\cdot), x>0
\end{array} .\right.\right.\right.
$$

Here, the phases are labeled in such way that $f_{l, r}$ are functions having at most one extremum within $[0,1]$ which is a local maximum. Recall that we have $f_{l, r}(0)=0$ and also $f_{l}(1), f_{r}(1)$ coincide.

For $\varepsilon>0$, the weak traces of the total fluxes $f_{l, r}(s)-\varepsilon \lambda_{l, r}(s) \partial_{x} \pi_{l, r}(s)$ at $\{x=0\}$ in (1) should match in order to ensure the conservation of the two phases. Moreover, the phase pressures should be continuous as long as the phase is present on both sides of the interface [17,21]; this leads to the trace relation $\left.\tilde{\pi}_{l}\left(s\left(t, 0^{-}\right)\right) \cap \tilde{\pi}_{r}\left(s(t,)^{+}\right)\right) \neq \emptyset$ with multivalued $\tilde{\pi}_{l, r}$, as explained above. Existence of solutions for $\varepsilon>0$ is shown in [16] (see also [4]) and compactness of the resulting family of solutions $s^{\varepsilon}$ comes under the additional assumption of strict nonlinearity of $f_{l, r}$ (the general case can be treated via a more specific $B V_{l o c}$ argument, see [4, Appendix A.1]).

The limit $s$ of $\left(s^{\varepsilon}\right)_{\varepsilon}$ satisfies the Kruzhkov entropy inequalities [25] away from the interface:

$$
\partial_{t}(\phi(x)|s-\kappa|)+\partial_{x} \Phi(x ; s, \kappa) \leq 0 \quad \text { in } \quad \mathcal{D}^{\prime}((\mathbb{R} \backslash\{0\}) \times(0, T)),
$$


where $\phi(x)=\phi_{l} \mathbb{1}_{x<0}+\phi_{r} \mathbb{1}_{x>0}$ and $\Phi(x ; s, \kappa)=\Phi_{l}(s, \kappa) \mathbb{1}_{x<0}+\Phi_{r}(s, \kappa) \mathbb{1}_{x>0}, \Phi_{l, r}(\cdot, \kappa)$ being the Kruzhkov entropy-fluxes $\operatorname{sign}(\cdot-\kappa)\left(f_{l, r}(\cdot)-f_{l, r}(\kappa)\right)$. Further, due to the conservative weak formulation of the equation, the conservativity relation at $x=0$ passes to the limit and yields the Rankine-Hugoniot condition $f_{l}\left(s\left(t, 0^{-}\right)\right)=f_{r}\left(s\left(t, 0^{+}\right)\right.$) (for strictly nonlinear $f_{l, r}$ strong traces $s\left(t, 0^{ \pm}\right)$exist due to Kruzhkov inequalities (12); see [7] for more generality). While the relation connecting capillary pressures does not pass to the limit, the condition inherited as $\varepsilon \rightarrow 0$ can be described implicitly by the specific global adapted entropy inequality:

$$
\begin{gathered}
\partial_{t}(\phi(x)|s-c(x)|)-\partial_{x} \Phi(x ; s, c(x)) \leq 0 \quad \text { in } \mathcal{D}^{\prime}(\mathbb{R} \times(0, T)), \\
\text { with } c(x):=\left\{\begin{array}{c}
s_{l}^{\pi}, x<0 \\
s_{r}^{\pi}, x>0 .
\end{array}\right.
\end{gathered}
$$

Actually, the issue of interface coupling for the singular limit of (1) as $\varepsilon \rightarrow 0$ reduces to the selection of the couple $\left(s_{l}^{\pi}, s_{r}^{\pi}\right)$ called connection, as described in [4]. Two qualitatively different cases may occur:

(I) the fluxes are connected at the "optimal connection level":

$$
\begin{aligned}
f_{l}\left(s_{l}^{\pi}\right)=f_{r}\left(s_{r}^{\pi}\right)=\bar{F}^{\text {opt }} \text { where } \bar{F}^{\text {opt }} & =\min \left\{\max _{[0,1]} f_{l}, \max _{[0,1]} f_{r}\right\}, \\
\text { with, moreover, } \quad f_{l}^{\prime}\left(s_{l}^{\pi}\right) & \leq 0 \text { and } f_{r}^{\prime}\left(s_{r}^{\pi}\right) \geq 0 .
\end{aligned}
$$

In this case, the vanishing capillarity limits are the "optimal solutions" obtained by Kaasschieter [24]. One can observe that in this case, the pressures are not connected: $\pi_{l}\left(s_{l}^{\pi}\right) \neq \pi_{r}\left(s_{r}^{\pi}\right)$.

(II) both capillary pressures and fluxes are connected:

$$
\text { (16) holds, and } \tilde{\pi}_{l}\left(s_{l}^{\pi}\right) \cap \tilde{\pi}_{r}\left(s_{r}^{\pi}\right) \neq 0 \text {, and } f_{l}\left(s_{l}^{\pi}\right)=f_{r}\left(s_{r}^{\pi}\right)=\bar{F} \leq \bar{F}^{o p t} \text {. }
$$

Notice that in both cases (I) and (II) we have (16). Except for very specific configurations of fluxes $f_{l, r}$, the two inequalities are strict in the case (II), while only one of them is strict in the case (I). As a consequence, following the terminology of $[2,10]$ the connection $\left(s_{l}^{\pi}, s_{r}^{\pi}\right)$ consists in a strictly undercompressive discontinuity in case (II), while it is only marginally undercompressive in case (I).

Remark 2.1 The case (II) was judged exceptional and neglected in the pioneering work [24]; let us stress that both (I) and (II) can be relevant, depending on the shape of the nonlinearities $f_{l, r}$ and $\pi_{l, r}$. We refer to [4] for a detailed discussion, and to [13,14] for examples.

To be precise, if (II) is possible then $\left(s_{l}^{\pi}, s_{r}^{\pi}\right)$ obeys (II), so that (I) only occurs when (II) cannot be realized. In practice, finding $s_{l, r}^{\pi}$ corresponds to intersecting the graph of a strictly decreasing function with a non-decreasing graph. Hence the uniqueness of the intersection point in (II) follows, while (I) happens when existence fails. In addition, one can observe that among all undercompressive (in the above sense) shocks with connected fluxes, conditions (I),(II) minimize the gap $\left|\pi_{l}\left(s_{l}^{\pi}\right)-\pi_{r}\left(s_{r}^{\pi}\right)\right|$, which is an intuitively appealing feature. We refer to [4] for the study of elementary vanishing capillarity profiles that provides the justification of the selection rule (I),(II)

As shown in [4], the function $c(x)$ in (14) chosen according to (I),(II) appears as an explicit vanishing capillarity limit for (1), i.e., it is an admissible solution for a very special initial datum. It is remarkable that the knowledge of $\left(c_{l}^{\pi}, c_{r}^{\pi}\right)$ and the rigid structure of the semigroups of solutions of (1) permit to characterize vanishing capillarity limits for all initial data. Indeed, we have 
Theorem 2.1 For every initial datum $u_{0} \in L^{\infty}(\mathbb{R})$ there exists a unique $u \in C\left([0, T] ; L_{\text {loc }}^{1}(\mathbb{R})\right)$ weak solution of (1),(11) with $\varepsilon=0$ that satisfies the Kruzhkov entropy inequalities (12) away from the interface and the global adapted entropy inequality (13) with $s_{l, r}^{\pi}$ in (14) given by the selection rule (I),(II). Moreover, the limit, as $\varepsilon \rightarrow 0$, of solutions $u^{\varepsilon}$ of (1) with datum $u_{0}$ exists and coincides with $u$.

This means that the property of being vanishing capillarity limit (which is very difficult to check directly) is fully characterized by entropy and well-chosen adapted entropy inequalities.

It is easily seen that extending such analysis to the multi-dimensional situation does not seem possible with respect to the existing state of the art. But some insight can be carried from the one-dimensional situation to the general case. Specifically, let us insist on the role of the parameter $\bar{F}$ which takes values in the interval $\left[\bar{F}^{\text {bar }}, \bar{F}^{\text {opt }}\right]$. It provides the connection level, which turns out to be the most relevant physical parameter at the vanishing capillarity limit. While the value $\bar{F}$ in (II) indicates the level of the interface flux for the particular solution $c(x)$ given by (14), it is shown in [4] that $\bar{F}$ is the largest possible value of the interface flux $f_{l}\left(s\left(t, 0^{-}\right)\right)=f_{r}\left(s\left(t, 0^{+}\right)\right)$for any solution of our singular limit problem. Thus the optimal entropy solution corresponding to $\bar{F}^{o p t}$ and case (I) optimizes (maximizes) the capacity of the interface to conduct the flow of the phases; the barrier entropy condition corresponding to $\bar{F}^{\text {bar }}$ minimizes the flow, which can be seen as a model for the oil trapping phenomenon.

The value $\bar{F}$ depends on $f_{l, r}$ but also on $\pi_{l, r}$ that are formally absent from (1) when $\varepsilon=0$. Let us denote it $\bar{F}^{\pi}$, in order to stress this dependence. The value $\bar{F}^{\pi}$ is determined by an easy algorithm solving a monotone nonlinear equation on $[0,1]$. It can be seen as the physical parameter that records the action of the capillary diffusion at the interface between the two rocks. But $\bar{F}^{\pi}$ can also be used, in a strikingly simple way, in the numerical simulation in order to guarantee the convergence of approximations to the physically correct solution. Indeed, as explained in [4, Prop. 9] (see also [5]), it is enough to take the Godunov numerical flux $G$ suitable for the approximation of the optimal solution (see [1]) and consider

$$
\begin{aligned}
& F_{\text {int }}:\left(z_{l}, z_{r}\right) \mapsto \min \left\{\bar{F}^{\pi}, G\left(z_{l}, z_{r}\right)\right\}, \\
& \text { with the optimal flux } G:\left(z_{l}, z_{r}\right) \mapsto \min \left\{f_{l}\left(\min \left\{z_{l}, \bar{s}_{l}\right\}\right), f_{r}\left(\max \left\{\bar{s}_{r}, z_{r}\right\}\right)\right\}
\end{aligned}
$$

where $\bar{s}_{l, r}:=\operatorname{argmax}_{[0,1]} f_{l, r}$. Finally, let us stress that the flux limitation in the Godunov scheme can be achieved by a less explicit method that nevertheless has a clear numerical interpretation; we will use it thoughout Section 3. Denote by $G_{l, r}$ the Godunov fluxes (see, e.g., $[1,5,22]$ ) associated with $f_{l, r}$, respectively; they can be computed using $\bar{s}_{l, r}$.

Lemma 2.2 (see [4, Lem. 8 and Prop. 9]) Let $\left(z_{l}, z_{r}\right) \in[0,1]^{2}$, then the system

$$
\left\{\begin{array}{l}
\pi \in \tilde{\pi}_{l} \cap \tilde{\pi}_{r} \\
G_{l}\left(z_{l}, \pi_{l}^{-1}(\pi)\right)=G_{r}\left(\pi_{r}^{-1}(\pi), z_{r}\right)
\end{array}\right.
$$

admits at least one solution $\pi \in \mathbb{R}$. The value $F_{\text {int }}\left(z_{l}, z_{r}\right):=G_{l}\left(z_{l}, \pi_{l}^{-1}(\pi)\right)=G_{r}\left(\pi_{r}^{-1}(\pi), z_{r}\right)$ is defined uniquely by (19), furthermore, it coincides with the value computed by the explicit formula (18).

The lemma introduces an additional variable $\pi$ on the interface that serves to connect the pressures in the vanishing capillarity limit; while it is rather useless for the one-dimensional problem, the value $\pi$ will be instrumental in the multi-dimensional schemes of Section 3. Following closely the 
proof of [4, Prop.9] one finds the algorithm to determine the value $\pi$ without solving (19); but this is not our goal. Heading towards a simpler practical implementation, let us generalize (19) as follows.

Proposition 2.3 If the Godunov fluxes $G_{l, r}$ are replaced in the scheme of [4] (including equation (19) for $F_{\text {int }}$ ) by consistent with $f_{l, r}$ monotone Lipschitz continuous numerical fluxes $R_{l, r}$ (see, e.g., [22]), then the scheme still converges to the solution given by Theorem 2.1.

Proof: It is easily checked that $F_{\text {int }}(a, b)$ remains increasing in $a$ and decreasing in $b$. Thus the scheme is monotone, and due the the Crandall-Tartar lemma, discrete $L^{1}$ contraction inequalities follow. Therefore the convergence arguments as in [4, Prop. 6] apply provided we show that the solution (14) of equation (1) avec $\varepsilon=0$ is a limit of some solutions of the scheme. This is obvious in case (II) because (14) gives a stationary solution to the scheme, due to the consistency of fluxes $R_{l, r}$. In case (I), we use a numerical counterpart of the construction of [4, Prop. 3(ii)]: using the monotonicity of the numerical flux, one constructs an appropriate stationary numerical profile. E.g., the situation where $s_{l}^{\pi}=\bar{s}_{l}$ we take the value $\tilde{s}_{l}^{\pi} \in\left[0, \bar{s}_{l}\right]$ such that $\pi_{l}\left(\tilde{s}_{l}^{\pi}\right)=\pi_{r}\left(s_{r}^{\pi}\right)$. There exists a solution to the scheme

$$
u=\left(u_{i}\right)_{i=-\infty}^{0} \text { such that } u_{0}=\tilde{s}_{l}^{\pi}, R_{l}\left(u_{-1}, u_{0}\right)=f_{r}\left(s_{r}^{\pi}\right) \text { and } \forall i \leq-1 R_{l}\left(u_{i-1}, u_{i}\right)=R_{l}\left(u_{i}, u_{i+1}\right),
$$

that we complement by the constant values $u_{i}:=s_{r}^{\pi}$ for $i>0$. The profile is monotone in $i$ thanks to the monotonicity of $R_{l}$, hence there exists $\ell:=\lim _{i \rightarrow-\infty} u_{i}$. Due to the continuity of $R_{l}$, from the above scheme we obtain that $R_{l}(\ell, \ell)=\lim _{i \rightarrow-\infty} R_{l}\left(u_{i-1}, u_{i}\right)=f_{r}\left(s_{r}^{\pi}\right)$, which yields $\ell=s_{l}^{\text {opt }}$. By a simple scaling argument, $u_{h}(x)=\sum_{i \in \mathbb{Z}} u_{i} \mathbb{1}_{[(i-1) h, i h]}$ converges, as $h \rightarrow 0$, to the function (14).

Remark 2.2 A particular case of construction (19) of the interface fluxes appears, under the form

$$
F_{\text {int }}\left(z_{l}, z_{r}\right):=G_{l}\left(z_{l}, s\right)=G_{r}\left(s, z_{r}\right) \text { for some } s \in[0,1],
$$

in the early work of Chavent, Cohen and Jaffré [18]. The choice of numerical fluxes (20) means that connection of the saturation values is expected at the interface; that is, $\pi_{l} \equiv \pi_{r}$ should be assumed in our model. Therefore the so obtained solutions correspond to the mathematical vanishing viscosity method. Notice that (20) was also used by Diehl [20] to characterize vanishing viscosity limits for general conservation laws with discontinuous flux (see [20, Section 3]). Let us stress that if $\pi_{l} \not \equiv \pi_{r}$, the vanishing viscosity regularization of the Buckley-Leverett equation leads, in general, to non-physical solutions.

\section{Finite volume approximation at vanishing capillarity limit}

We propose three finite volume schemes designed for approximating problem (3), (5), (6), (8)-(10). The first one is described in Section 3.2. It is obtained by neglecting the inner capillary diffusion in the scheme proposed in [8], which yields a fully implicit (and thus fully coupled) scheme whose unknowns are the saturations $\left(s_{K}^{n+1}\right)$ and the pressures $\left(P_{K}^{n+1}\right)$ in the control volumes as well as the capillary pressure transmission parameters $\left(\pi_{\sigma}^{n+1}\right)$ on the edges $\sigma$ lying in the inner interface $\Gamma$. The main advantage of this scheme is that it preserves energy bounds, ensuring some stability. 
Its main drawback is of course its computational cost, that we aim to reduce in the variants of the scheme. The first alternative we propose in Section 3.3 consists in the IMPES scheme decoupling the saturations $\left(s_{K}^{n+1}\right)$ from the pressure and capillary pressure unknowns $\left(P_{K}^{n+1}\right)$ and $\left(\pi_{\sigma}^{n+1}\right)$. Further, in Section 3.4 we propose an algorithm that fully decouples the saturations $\left(s_{K}^{n+1}\right)$, the pressures $\left(P_{K}^{n+1}\right)$ and the interface capillary pressures $\left(\pi_{\sigma}^{n+1}\right)$.

For the sake of simplicity, we suppose in the sequel that the porous medium is isotropic, i.e. $\underline{\mathbf{K}}_{i}=k_{i} \underline{\mathbf{I}}_{d}$, so that the elliptic equation (3) can be approximated by means of a two-point flux FV-scheme. The consistency of such a method requires the so-called orthogonality condition on the mesh that is recalled in Section 3.1. The natural extensions to the case of anisotropic porous media and to more general meshes are not discussed in this paper.

\subsection{Discretization of $\Omega \times(0, T)$}

Since the scheme used for discretizing the elliptic equation is based on a two-point flux approximation, the space discretization has to fulfill some admissibility condition. The notion of admissible discretization of $\Omega$ is inspired from the one given in [22], and additionally requires that each cell $K$ is either in $\Omega_{1}$ or in $\Omega_{2}$. We note $\mathcal{T}_{i}$ the set of all the cells included in $\Omega_{i}$, so that $\bar{\Omega}_{i}=\bigcup_{K \in \mathcal{T}_{i}} \bar{K}_{i}$. As a consequence, the interface $\Gamma$ is made of a union of a subset of edges denoted by $\mathcal{E}_{\Gamma}$, i.e. $\Gamma=\bigcup_{\sigma \in \mathcal{E}_{\Gamma}} \bar{\sigma}$. For each cell $K \in \mathcal{T}$, there is a point $x_{K} \in K$ called center of $K$ such that, denoting by $K \mid L$ the edge between $K$ and its neighboring cell $L$, the straight line $\left(x_{K} x_{L}\right)$ is orthogonal to $K \mid L$. We refer to [8, Definition 2.1] for a complete definition of the notion of admissible discretization of $\Omega$ that we use here.

The size of the mesh is $h=\max _{K \in \mathcal{T}} \operatorname{diam}(K)$; we ask the usual regularity assumption for a family of meshes, namely, that there exist $\alpha>0$ such that $|K| \geq \alpha h^{d},|\partial K|=\sum_{\sigma \in \mathcal{E}_{K}}|\sigma| \leq \frac{1}{\alpha} h^{d-1}$ for $K \in \mathcal{T}$. The time discretization consists of a sequence of time steps $\left.\left(\Delta t^{n}\right)\right)_{0 \leq n \leq N}$ which have to fulfill a CFL condition when explicit schemes are considered. We set $t^{0}=0, t^{n+1}=t^{n}+\Delta t^{n}$ and assume $t^{N}=T$.

\subsection{The coupled implicit scheme}

The scheme proposed now consists in fully neglecting the capillary diffusion (that is, merely setting $\varepsilon=0$ ) in the scheme proposed and validated in [8] for $\varepsilon>0$. Let us stress that for the onedimensional case, the scheme of [8] is asymptotic-preserving, i.e., as $\varepsilon \rightarrow 0$ it converges a numerical scheme that does approximate the entropy solution described in Section 2 (see [4, Cor. 10]).

The first equation of (3) is approximated by

$$
\sum_{\sigma \in \mathcal{E}_{K}}|\sigma| u_{K, \sigma}^{n+1}=|K| q_{o}\left(s_{K}^{n+1}\right)+|K| q_{w}\left(s_{K}^{n+1}\right) \quad \forall K \in \mathcal{T}
$$

where $u_{\underline{\mathbf{K}}, \sigma}^{n}$ is an approximation of $\frac{1}{\Delta t^{n}|\sigma|} \int_{t^{n}}^{t^{n+1}} \int_{\sigma} \mathbf{u}_{\mathbf{t}} \cdot \mathbf{n}_{K, \sigma} \mathrm{d} x \mathrm{~d} t$, where $n_{K, \sigma}$ denotes the normal to $\sigma$ outward w.r.t. $K$. The source term $q_{o}$ is supposed to be decreasing and such that $q_{o}(0)=0$, while the source term $q_{w}$ is supposed to be increasing and such that $q_{w}(1) \geq 0$. The quantity $u_{K, \sigma}^{n+1}$ is given by

$$
u_{K, \sigma}^{n+1}=0 \text { if } \sigma \subset \partial \Omega, \quad u_{K, \sigma}^{n+1}=k_{i}\left(M_{K \mid L}^{n+1} \frac{P_{K}^{n+1}-P_{L}^{n+1}}{d\left(x_{K}, x_{L}\right)}+\zeta_{K \mid L}^{n+1}\right) \text { if } \sigma=K \mid L \in \mathcal{E}_{i},
$$


where $M_{K \mid L}^{n+1}=\frac{M_{K}\left(s_{K}^{n+1}\right) M_{L}\left(s_{L}^{n+1}\right) d\left(x_{K}, x_{L}\right)}{M_{K}\left(s_{K}^{n+1}\right) d\left(x_{K}, \sigma\right)+M_{L}\left(s_{L}^{n+1}\right) d\left(x_{L}, \sigma\right)}$ and $\zeta_{K, L}^{n+1}$ denotes the Rusanov numerical flux for the flux function $\zeta_{i}(s) \mathbf{g} \cdot \mathbf{n}_{K, \sigma}$ and for the Riemann problem with initial data $s_{K}^{n+1} \mathbb{1}_{x<0}+s_{L}^{n+1} \mathbb{1}_{x>0}$. The case $\sigma=K \mid L \in \mathcal{E}_{\Gamma}$ is treated by means of the introduction of two new unknowns $P_{K, \sigma}^{n+1}$ and $P_{L, \sigma}^{n+1}$ to enforce the continuity of the flux (4) and the pressure condition (5) at the discrete level. Denoting by $g_{K, \sigma}=\mathbf{g} \cdot \mathbf{n}_{K, \sigma}$, we prescribe at the interface that for all $\sigma=K \mid L \in \mathcal{E}_{\Gamma}$,

$$
u_{K, \sigma}^{n+1}+u_{L, \sigma}^{n+1}=0 \text { with } u_{J, \sigma}^{n+1}=k_{J}\left(M_{J}\left(s_{J}^{n+1}\right) \frac{P_{J}^{n+1}-P_{J, \sigma}^{n+1}}{d\left(x_{J}, \sigma\right)}+\zeta_{J}\left(s_{J}^{n+1}\right) g_{J, \sigma}\right), \quad J=K, L .
$$

Moreover, the interface pressures $P_{K, \sigma}^{n+1}$ and $P_{L, \sigma}^{n+1}$ have to fulfill

$$
P_{K, \sigma}^{n+1}-Z_{K}\left(\pi_{\sigma}^{n+1}\right)=P_{L, \sigma}^{n+1}-Z_{L}\left(\pi_{\sigma}^{n+1}\right),
$$

where the unknowns $\left(\pi_{\sigma}^{n+1}\right)_{\sigma \in \mathcal{E}_{\Gamma}}$ are used to ensure the pressure transmission. Notice that for every $\sigma \in \mathcal{E}_{\Gamma}$, unknowns $P_{K, \sigma}^{n+1}$ and $P_{L, \sigma}^{n+1}$ are readily eliminated thanks to the linear relations (23)-(24). by

The initial datum $s_{0}$ is discretized by setting $s_{K}^{0}=\frac{1}{|K|} \int_{K} s_{0}(x) \mathrm{d} x$. Equation (8) is discretized

$$
\begin{gathered}
\frac{s_{K}^{n+1}-s_{K}^{n}}{\Delta t^{n}}|K|+\sum_{\sigma \in \mathcal{E}_{K}}|\sigma| F_{K, \sigma}^{n+1}=|K| q_{o}\left(s_{K}^{n+1}\right), \\
F_{K, \sigma}^{n+1}=0 \text { if } \sigma \subset \partial \Omega, \quad F_{K, \sigma}^{n+1}=\mathcal{R}\left(\mathcal{F}_{K, \sigma}^{n+1} ; s_{K}^{n+1}, s_{L}^{n+1}\right) \text { if } \sigma=K \mid L \in \mathcal{E}_{i},
\end{gathered}
$$

where

$$
\mathcal{F}_{K, \sigma}^{n+1} \text { denotes the function } s \mapsto f_{K}(s) u_{K, \sigma}^{n+1}+k_{K} \rho \lambda_{K}(s) g_{K, \sigma}
$$

and where $\mathcal{R}\left(\mathcal{F} ; s_{L}, s_{R}\right)$ denotes the Rusanov flux corresponding to the flux function $\mathcal{F}$ and to left and right states given by $s_{L}, s_{R}$. To handle the fluxes across the interfaces $\sigma \in \mathcal{E}_{\Gamma}$, additional unknowns $\pi_{\sigma}^{n+1}$ introduced in (24) are exploited. Namely, for $\sigma=K \mid L \in \mathcal{E}_{\Gamma}$, the flux $F_{K, \sigma}^{n+1}$ is defined by

$$
F_{K, \sigma}^{n+1}+F_{L, \sigma}^{n+1}=0 \text { where for } J=K \text { and } J=L, F_{J, \sigma}^{n+1}=\mathcal{R}\left(\mathcal{F}_{J, \sigma}^{n+1} ; s_{J}^{n+1}, \tilde{\pi}_{J}^{-1}\left(\pi_{\sigma}^{n+1}\right)\right) .
$$

Relations (27) yield one equation per unknown $\pi_{\sigma}^{n+1}, \sigma \in \mathcal{E}_{\Gamma}$, thus the system is closed. Notice that (24),(27) are directly inspired by the approach (19) of the one-dimensional case, but where the Godunov flux has been replaced by the Rusanov flux by virtue of Proposition 2.3.

The main advantage of this approximation is that it is unconditionally stable with respect to the time discretization. The following proposition can be proved by following the method proposed in $[8]$.

Proposition 3.1 For all admissible discretization of $\Omega$, and for all $n \in\{0, \ldots, N-1\}$, there exists $\left(\left(s_{K}^{n+1}\right)_{K \in \mathcal{T}},\left(P_{K}^{n+1}\right)_{K \in \mathcal{T}},\left(\pi_{\sigma}^{n+1}\right)_{\sigma \in \mathcal{E}_{\Gamma}}\right) \in[0,1]^{\# \mathcal{T}} \times \mathbb{R}^{\# \mathcal{T}} \times \overline{\mathbb{R}}^{\# \mathcal{E}_{\Gamma}}$ solution to the scheme $(21)-(27)$.

It is possible, following the sketch of the proof of Proposition 3.5 in [8], to derive the following estimate on the discrete $L^{2}\left((0, T) ; H^{1}\left(\Omega_{i}\right)\right)$ semi-norm (cf. [21,22]):

$$
\sum_{i=1,2} \sum_{n=0}^{N-1} \Delta t^{n}\left(\sum_{\sigma=K \mid L \in \mathcal{E}_{i}}|\sigma| \frac{\left(P_{K}^{n+1}-P_{L}^{n+1}\right)^{2}}{d\left(x_{K}, x_{L}\right)}\right) \leq C
$$


From this estimate, we can prove that the piecewise constant approximate pressure $P_{\mathcal{D}}$ converges weakly in $L^{2}\left(\Omega_{i} \times(0, T)\right)$ towards some pressure $P \in L^{2}\left((0, T) ; H^{1}\left(\Omega_{i}\right)\right)$. We also deduce from the fact that $0 \leq s_{K}^{n+1} \leq 1$ for all $K \in \mathcal{T}$ that the piecewise constant approximate saturation $s_{\mathcal{D}}$ converges in the $L^{\infty}(\Omega \times(0, T))$ weak- $\star$ sense towards a function $s$ in $L^{\infty}(\Omega \times(0, T))$, with $0 \leq s \leq 1$ a.e. in $\Omega \times(0, T)$. This is obviously not sufficient to pass to the limit in the nonlinearities if the problem. Nevertheless, a complete convergence study of the scheme presented above has been performed in [4] in the one-dimensional framework, where it was possible to establish rigorous convergence results.

\subsection{The $(P, \pi)-s$ partially decoupled explicit scheme}

As it will appear in the Section 4 where the numerical results are exhibited, the scheme proposed in Section 3.2 is quite expensive due to the fact that it is fully coupled and implicit. Our goal is then to uncouple some discrete equations, introducing an IMPES (IMplicit Pressure - Explicit Saturation) discretization of equations (3), (5), (6), (8)-(10). To do so, given $\left(s_{K}^{n}\right)_{K \in \mathcal{T}}$, we compute first the pressure variables $\left(\left(P_{K}^{n+1}\right)_{K \in \mathcal{T}},\left(\pi_{\sigma}^{n+1}\right)_{\sigma \in \mathcal{E}_{\Gamma}}\right)$, and then we use these new values of the pressures to compute $\left(s_{K}^{n+1}\right)_{K \in \mathcal{T}}$ by means of an explicit finite volume scheme.

a) Pressure step. We assume that $\left(s_{K}^{n}\right)_{K \in \mathcal{T}}$ is given in $[0,1]^{\# \mathcal{T}}$. The system we intend to solve is then

$$
\begin{gathered}
\sum_{\sigma \in \mathcal{E}_{K}}|\sigma| u_{K, \sigma}^{n+1}=|K| q_{o}\left(s_{K}^{n}\right)+|K| q_{w}\left(s_{K}^{n}\right) \quad \forall K \in \mathcal{T}, \\
u_{K, \sigma}^{n+1}=0 \text { if } \sigma \subset \partial \Omega, \quad u_{K, \sigma}^{n+1}=k_{i}\left(M_{K \mid L}^{n} \frac{P_{K}^{n+1}-P_{L}^{n+1}}{d\left(x_{K}, x_{L}\right)}+\zeta_{K \mid L}^{n}\right) \quad \text { if } \sigma=K \mid L \in \mathcal{E}_{i}, \\
u_{K, \sigma}^{n+1}+u_{L, \sigma}^{n+1}=0, u_{J, \sigma}^{n+1}=k_{J}\left(M_{J}\left(s_{J}^{n}\right) \frac{P_{J}^{n+1}-P_{J, \sigma}^{n+1}}{d\left(x_{J}, \sigma\right)}+\zeta_{J}\left(s_{J}^{n}\right) g_{J, \sigma}\right), J=K, L, \text { if } \sigma \in \mathcal{E}_{\Gamma} .
\end{gathered}
$$

while equation (24) still has to hold. After eliminating $P_{K, \sigma}^{n+1}$ and $P_{L, \sigma}^{n+1}$ thanks to (24) and (31), we obtain a system of $\# \mathcal{T}$ equations (29) for the $\# \mathcal{T}+\# \mathcal{E}_{\Gamma}$ unknowns $\left(P_{K}^{n+1}\right)_{K \in \mathcal{T}},\left(\pi_{\sigma}^{n+1}\right)_{K \in \mathcal{T}}$. In order to close the system we impose that, for all $\sigma \in \mathcal{E}_{\Gamma}$,

$$
F_{K, \sigma}^{n}+F_{L, \sigma}^{n}=0 \quad \text { where for } J=K \text { and } J=L, \quad F_{J, \sigma}^{n}=\mathcal{R}\left(\mathcal{F}_{J, \sigma}^{n+1} ; s_{J}^{n}, \tilde{\pi}_{J}^{-1}\left(\pi_{\sigma}^{n+1}\right)\right) .
$$

Note that if $u_{K, \sigma}^{n+1}$ is given, then equation (32) has a solution $\pi_{\sigma}^{n+1}$ thanks to a version of Lemma 2.2.

Proposition 3.2 Given $\left(s_{K}^{n}\right)_{K \in \mathcal{T}} \in[0,1]^{\# \mathcal{T}}$, there exists a solution $\left(\left(P_{K}^{n+1}\right)_{K \in \mathcal{T}},\left(\pi_{\sigma}^{n+1}\right)_{\sigma \in \mathcal{E}_{\Gamma}}\right)$ belonging to $\mathbb{R}^{\# \mathcal{T}} \times \overline{\mathbb{R}}^{\# \mathcal{E}_{\Gamma}}$ to the problem (24), (29)-(32).

b) Saturation step. This step consists in deducing $\left(s_{K}^{n+1}\right)_{K \in \mathcal{T}}$ from $\left(s_{K}^{n}\right)_{K \in \mathcal{T}}$ and the freshly computed pressures $\left(P_{K}^{n+1}\right)_{K \in \mathcal{T}},\left(\pi_{\sigma}^{n+1}\right)_{\sigma \in \mathcal{E}_{\Gamma}}$. This is done by means of an explicit finite volume scheme.

Equation (8) is now discretized by

$$
\frac{s_{K}^{n+1}-s_{K}^{n}}{\Delta t^{n}}|K|+\sum_{\sigma \in \mathcal{E}_{K}}|\sigma| F_{K, \sigma}^{n}=|K|\left(-L_{q_{o}} s_{K}^{n+1}+q_{o}\left(s_{K}^{n}\right)+L_{q_{o}} s_{K}^{n}\right),
$$


where $L_{q_{o}}$ denotes the Lispchitz constant of $q_{o}$, and where

$$
F_{K, \sigma}^{n}=0 \text { if } \sigma \subset \partial \Omega, \quad F_{K, \sigma}^{n}=\mathcal{R}\left(\mathcal{F}_{K, \sigma}^{n+1} ; s_{K}^{n}, s_{L}^{n}\right) \text { if } \sigma=K \mid L \in \mathcal{E}_{i}
$$

(in addition, recall that the fluxes $F_{K, \sigma}^{n}$ for $\sigma \in \mathcal{E}_{\Gamma}$ have already been computed during the pressure step in (32)). A CFL condition is mandatory to ensure stability of the scheme at this step. Denoting by $L_{f_{i}}$ (resp. $\left.L_{\lambda_{i}}\right)$ the Lispchitz constant of $f_{i}\left(\right.$ resp. $\left.\lambda_{i}\right)$, then we require that

$$
\Delta t^{n} \leq \alpha^{2} h\left(\max _{i \in\{1,2\}} L_{f_{i}} \max _{\sigma \in \mathcal{E}}\left|u_{K, \sigma}^{n+1}\right|+|\rho||\mathbf{g}| \max _{i \in\{1,2\}} L_{\lambda_{i}}\right) .
$$

Proposition 3.3 Given $\left(s_{K}^{n}\right)_{K \in \mathcal{T}} \in[0,1]^{\# \mathcal{T}}$, and given $\left(\left(P_{K}^{n+1}\right)_{K \in \mathcal{T}},\left(\pi_{\sigma}^{n+1}\right)_{\sigma \in \mathcal{E}_{\Gamma}}\right) \in \mathbb{R}^{\# \mathcal{T}} \times \overline{\mathbb{R}}^{\# \mathcal{E}_{\Gamma}}$, under the CFL condition (35), for all $K \in \mathcal{T}$ one has $0 \leq s_{K}^{n+1} \leq 1$.

The above result follows from the fact that equation (33) can be rewritten as

$$
s_{K}^{n+1}=s_{K}^{n}-\frac{\Delta t^{n}}{\left(1+L_{q_{o}}\right)|K|} \sum_{\sigma \in \mathcal{E}_{K}}|\sigma| F_{K, \sigma}^{n}+\Delta t^{n} \frac{q_{o}\left(s_{K}^{n}\right)}{\left(1+L_{q_{0}}\right)},
$$

the right-hand side of the above expression being a nondecreasing function with respect to (w.r.t.) each of the variables $s_{K}^{n}, K \in \mathcal{T}$. Notice that the interface fluxes $F_{K, \sigma}^{n}$ given by (32) depend in a monotone and Lipschitz continuous way on $s_{K}^{n}$ and $s_{L}^{n}$ because, thanks to Lemma 2.2, they can be rewritten under the form (18) on which monotonicity and Lipschitz continuity is obvious. Remark also that the discretization of the source term in (33) is such that it is linear w.r.t. $s_{K}^{n+1}$ (thus the scheme remains explicit), and so that it is increasing w.r.t. $s_{K}^{n+1}$ and nonincreasing w.r.t. $s_{K}^{n}$. This yields a stabilization of the scheme in the sense that the CFL condition (35) does not depend on the source term $q_{o}$.

\subsection{The fully decoupled explicit scheme}

Notice that in Pressure Step of the above IMPES scheme, relations (24) and (32) involving $\left(\pi_{\sigma}^{n+1}\right)_{\sigma \in \mathcal{E}_{G}}$ are fully coupled. In particular, the expressions of the flux functions $\mathcal{F}_{K, \sigma}^{n+1}$ needed to compute $F_{K, \sigma}^{n}$ depend on the whole set of values of $\left(\pi_{\sigma}^{n+1}\right)_{\sigma \in \mathcal{E}_{G}}$ via the values $u_{K, \sigma}^{n+1}$. Here, we propose a variant of the previous scheme where the computation of the capillary pressure transmission parameters $\left(\pi_{\sigma}^{n+1}\right)_{\sigma \in \mathcal{E}_{\Gamma}}$ is decoupled from the computation of the global pressures $\left(P_{K}^{n+1}\right)_{K \in \mathcal{T}}$.

For this scheme, we need to initialize the capillary pressure. To do so, we build $\pi_{\sigma}^{0}$ as the linear interpolation between the neighboring cell values of the capillary pressure:

$$
\pi_{\sigma}^{0}=\frac{\pi_{K}\left(s_{K}^{0}\right) d\left(x_{L}, \sigma\right)+\pi_{L}\left(s_{L}^{0}\right) d\left(x_{K}, \sigma\right)}{d\left(x_{K}, x_{L}\right)}, \quad \forall \sigma=K \mid L \in \mathcal{E}_{\Gamma} .
$$

a) Global pressure step. Values $\left(s_{K}^{n}\right)_{K \in \mathcal{T}}$ and $\left(\pi_{\sigma}^{n}\right)_{\sigma \in \mathcal{E}_{\Gamma}}$ being given, we obtain $\left(P_{K}^{n+1}\right)_{K \in \mathcal{T}}$ as solution to (29)-(31), closed by the interface relation

$$
P_{K, \sigma}^{n+1}-Z_{K}\left(\pi_{\sigma}^{n}\right)=P_{L, \sigma}^{n+1}-Z_{L}\left(\pi_{\sigma}^{n}\right), \quad \forall \sigma \in \mathcal{E}_{\Gamma} .
$$

This system is linear and one can check that it admits a unique solution $\left(P_{K}^{n+1}\right)_{K \in \mathcal{T}} \in \mathbb{R}^{\# \mathcal{T}}$. 
b) Capillary pressure step. The previous step also yields the values $u_{K, \sigma}^{n+1}$, thus the expressions of the fluxes $\mathcal{F}_{K, \sigma}^{n+1}$ are made available. Now we can compute the values $\pi_{\sigma}^{n+1}$ for each $\sigma \in \mathcal{E}_{\Gamma}$ by solving

$$
F_{K, \sigma}^{n}+F_{L, \sigma}^{n}=0 \text { with } F_{J, \sigma}^{n}=\mathcal{R}\left(\mathcal{F}_{J, \sigma}^{n+1} ; s_{J}^{n}, \tilde{\pi}_{J}^{-1}\left(\pi_{\sigma}^{n+1}\right)\right) \text { for } J=K, L
$$

(we have one nonlinear equation per edge $\sigma \in \mathcal{E}_{\Gamma}$, solved separately for each $\sigma$ ). Existence of a solution $\pi_{\sigma}^{n+1}$ and uniqueness of the resulting fluxes $F_{K, \sigma}^{n}$ follow by a monotonicity argument, as for Lemma 2.2.

c) Saturation step. The saturations $\left(s_{K}^{n+1}\right)_{K \in \mathcal{T}}$ are computed explicitly by equations (33) and the fluxes readily computed with (38) and (34).

\section{$4 \quad$ Numerical experiments}

All the three schemes of Section 3 preserve the physical bounds on the saturation $s$. It seems much more delicate to achieve stability estimates like (28), especially for the fully decoupled scheme, and convergence analysis is beyond our reach. Therefore we now switch to numerical tests. Below, the fully decoupled scheme is used in all the test cases; the fully implicit and the IMPES schemes actually yield quite close numerical solutions but a longer computational time.

\subsection{Comparison with 1D reference solution obtained by a convergent scheme.}

Let $\Omega=(-1,1)^{2}$ be 2 -dimensional domain composed of two horizontal layers $\Omega_{1}=\{(x, y) \in \Omega \mid y<$ $0\}$ and $\Omega_{2}=\{(x, y) \in \Omega \mid y>0\}$, which corresponds to different rock-types. We prescribe a zero total flux on the whole boundary $\partial \Omega$. The boundary conditions for the saturation equation are given by

$$
\begin{array}{lll}
s=0.5 & \text { on } & \Gamma_{1}=\{(x, y) \in \partial \Omega \text { s.t. }|y|=1\}, \\
\left(\mathbf{u}_{\mathrm{t}} f_{i}(s)+\lambda_{i}(u) \underline{\mathbf{K}}_{i} \mathbf{g}\right) \cdot \mathbf{n}=0 & \text { on } & \Gamma_{2}=\{(x, y) \in \partial \Omega \text { s.t. }|x|=1\},
\end{array}
$$

the initial saturation is set to $s_{0}=0.5$. We set $\mathbf{g}=-9.81 \mathbf{e}_{\mathbf{y}}, \mu_{o}=1, \mu_{w}=3, \rho_{o}=0.87, \rho_{w}=1$, and

$$
f=\frac{s}{s-\mu_{o} / \mu_{w}(1-s)}, \quad \lambda=\left(\rho_{o}-\rho_{w}\right) \frac{s(1-s)}{\mu_{w} s-\mu_{o}(1-s)}, \quad \zeta=\frac{\rho_{o}}{\mu_{o}} s+\frac{\rho_{w}}{\mu_{o}}(1-s)
$$

The porosity $\phi$ is constant and set to $\phi=1$; the absolute permeability $\underline{\mathbf{K}}$ is given by $\underline{\mathbf{K}}_{1}=10$, $\underline{\mathbf{K}}_{2}=5$.

We take capillary pressure curves $\pi_{i}(s):=\pi_{i, 0}-\ln (1-s)$ that differ only by the value of the entry pressure: $\pi_{1,0}$ is set to 0 , while $\pi_{2,0}$ takes values 0.5 (Test 1a) or 2 (Test $1 \mathrm{~b}$ ). The flow is driven by buoyancy and by the spatial discontinuity: roughly speaking, the second rock has a smaller pores, so that it is more attractive for water. The total flow $\mathbf{u}_{t}$ being zero, the oil phase is displaced from the rock $\Omega_{2}$.

This is actually the 1D test borrowed from [4]; there exists an $x$-independent solution. However, using triangular meshes we ensure that the scheme we test is truly 2D. Indeed, discrete solution is computed by the fully decoupled IMPES scheme on structured triangular meshes with different number of volumes. It is compared to the reference 1D numerical solution evaluated using 1000 grid 
points (this is indeed a reference solution, due to Th. 2.1 and Prop. 2.3). We present the results in Fig. 1 (Test 1a), Fig. 2 (Test 1b). In each case, 2D schemes for two different choices of numerical fluxes (namely the Godunov and the Rusanov ones) are compared. For the final time $T=0.2$, the error in the norm $L^{2}((0, T) \times \Omega)$ is plotted at the right of Fig. 1,2. We observe convergence rates close to 1 (for the Godunov flux) and 0.5 (the Rusanov flux). Notice that in Test 1a the two discretizations exhibit quite different behavior. In fact, for both choices of fluxes the connecting capillary pressure $\pi$ is such that $\pi_{2}^{-1}(\pi)>0$. Thus, a small amount of oil propagating in the domain $\Omega_{2}$ is due only to the extra diffusion introduced by Rusanov flux. This is not the case for the Godunov flux, which provides the exact solution to the Riemann problem.
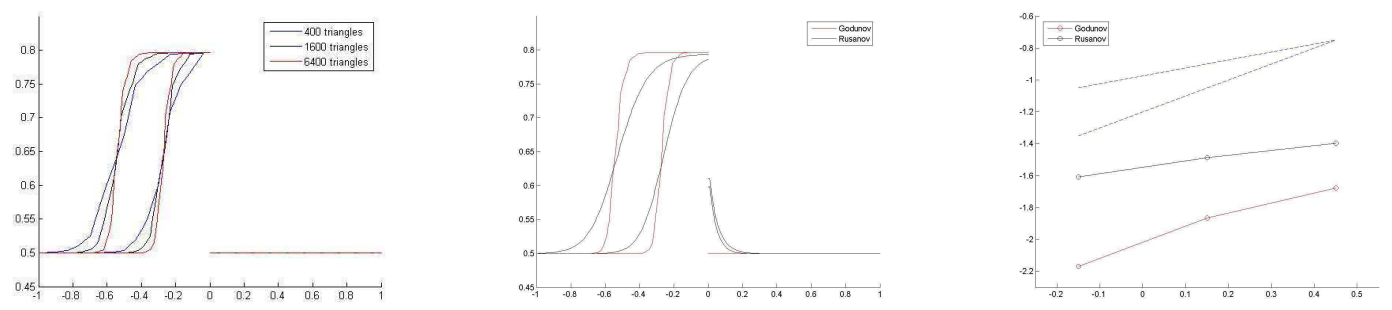

Figure 1: Test 1a (left to right): Oil saturation profile at $t=0.1$ and $t=0.2$ (Godunov flux); Godunov/Rusanov comparison; Error in $L^{2}$, red for Godunov (the bottom line) and black for Rusanov; dashed lines: slopes 1 and $1 / 2$.
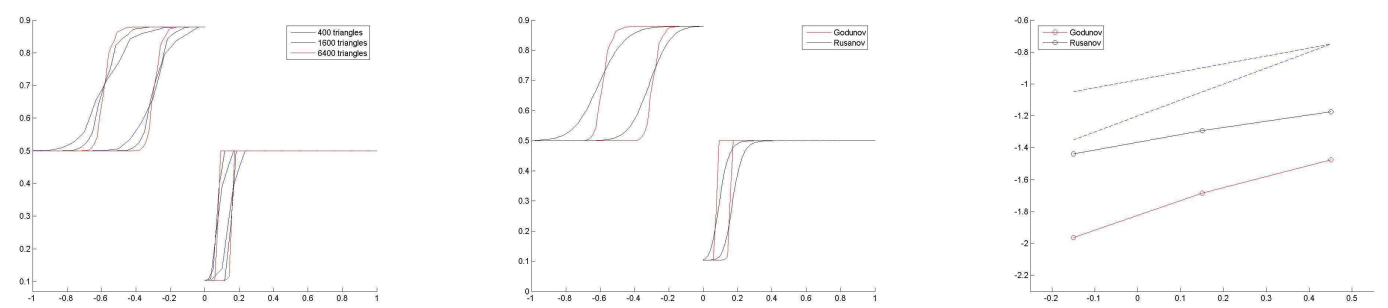

Figure 2: Test $\mathbf{1 b}$ (left to right): Oil saturation profile at $t=0.1$ and $t=0.2$ (Godunov flux); Godunov/Rusanov comparison; Error in $L^{2}$, red for Godunov (bottom line) and in black for Rusanov; dashed lines: slopes 1 and $1 / 2$.

\subsection{A 2D flow constrained by capillary barriers}

We now turn to a truly $2 D$ test case inspired from [23, test case 4.3]. The $2 D$ domain $\Omega$ (see Fig. 3, top left), which mostly consists of rock $\Omega_{1}$, is initially saturated in water: $s_{0}=0$. The flow is constrained by a presence of two barriers (rock $\left.\Omega_{2}\right)$ having a higher entry pressure $\left(\pi_{0,1}=0\right.$, $\left.\pi_{0,2}=1.5\right)$. The vertical boundaries are assumed to be impermeable, so on $\Gamma_{N}$ zero flux is prescribed for both phases. At the bottom and the top of $\Omega$ we prescribe a constant rate of a total flux, i.e

$$
\mathbf{u}_{\mathrm{t}} \cdot \mathbf{n}=-1 \quad \text { on } \Gamma_{\text {in }}, \quad \mathbf{u}_{\mathrm{t}} \cdot \mathbf{n}=1 \quad \text { on } \Gamma_{\text {out }} .
$$


The constant saturation value $s=0.5$ is imposed on $\Gamma_{i n}$. We use the same set of data as previously, except for the absolute permeability $\underline{\mathbf{K}}_{1,2}$ now assumed to be equal to 1 in both sub-domains. We use here the Rusanov scheme as an approximate Riemann solver.
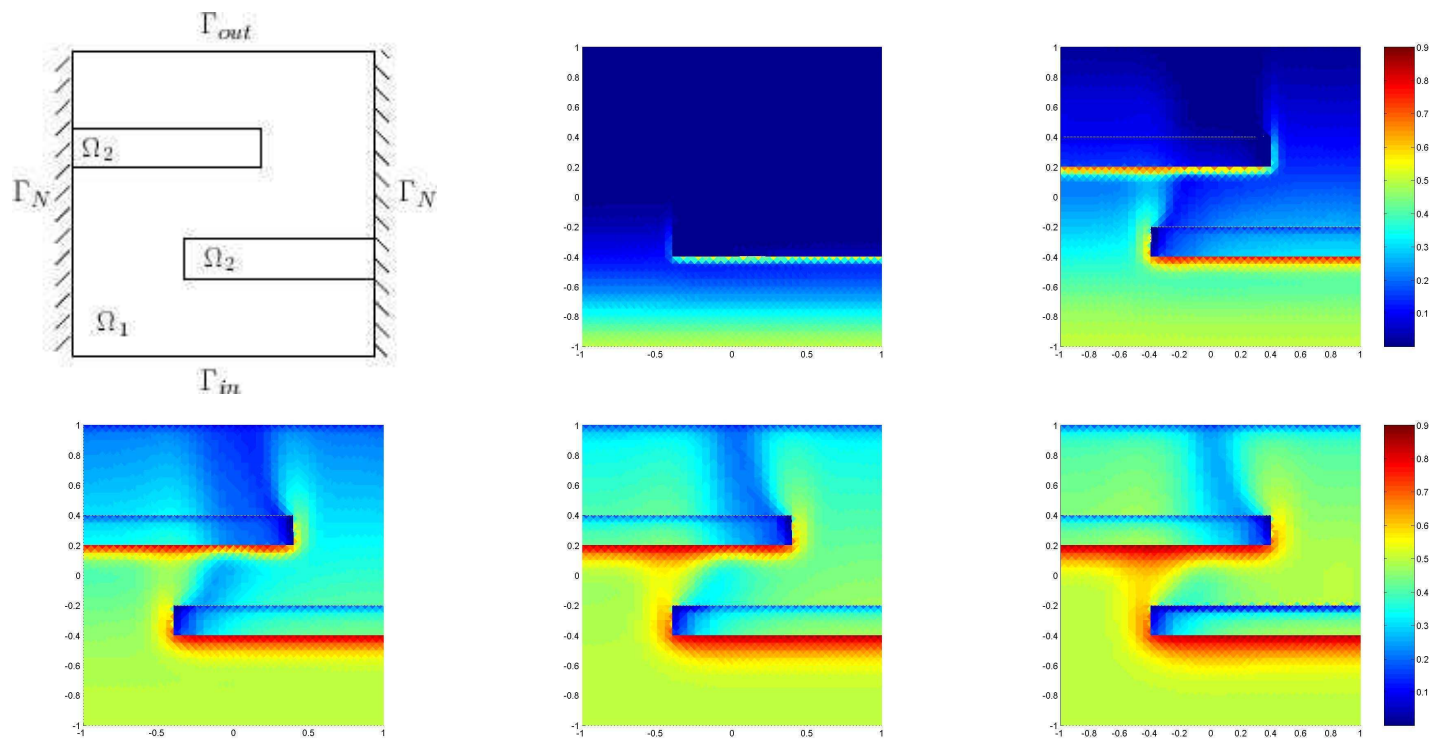

Figure 3: Test 2. Computational domain and the oil saturation field at time $t=$ $0.075,0.2,0.4,0.6,0.8$.

Even if both rocks $\Omega_{i}$ have the same intrinsic permeability (so that the flux function is continuous across the interface), it appears that some oil remains trapped under the capillary barriers. Indeed, due to singular capillary effects at rock discontinuities, the relevant solution is not the usual entropy solution [4]. A flux limitation [6] is active at the lower boundary of the barrier, hampering the progression of the oil phase. This singular effect depends on the capillary pressure functions $\pi_{i}$, despite the capillary diffusion has been neglected within the rocks $\Omega_{i}$.

Acknowledgement. The work of the first author was partially supported by the French ANR project CoToCoLa. The work of the second and third authors was partially supported by GNR MoMaS, CNRS-2439 (PACEN/CNRS, ANDRA, BRGM, CEA, EDF, IRSN).

\section{References}

[1] Adimurthi, J. Jaffré, and G. D. Veerappa Gowda. Godunov-type methods for conservation laws with a flux function discontinuous in space. SIAM J. Numer. Anal., 42(1): 179-208, 2004 .

[2] Adimurthi, S. Mishra, and G. D. Veerappa Gowda. Optimal entropy solutions for conservation laws with discontinuous flux-functions. J. Hyperbolic Differ. Equ., 2(4): 783-837, 2005. 
[3] B. Amaziane, J.P. Milisic, M. Panfilov and L. Pankratov. Generalized nonequilibrium capillary relations for two-phase flow through heterogeneous media. Phys. Rev. E, 85(1): 1-18, 2012 (published electronically).

[4] B. Andreianov and C. Cancès. Vanishing capillarity solutions of Buckley-Leverett equation with gravity in two-rocks' medium. Comput. Geosci., to appear.

[5] B. Andreianov and C. Cancès. The Godunov scheme for scalar conservation laws with discontinuous bell-shaped flux functions. Appl. Math. Letters, 25: 1844-1848, 2012.

[6] B. Andreianov, P. Goatin and N. Seguin. Finite volume schemes for locally constrained conservation laws. Numer. Math., 115(4): 609-645, 2010.

[7] B. Andreianov, K. Karlsen, and N. Risebro. A theory of $L^{1}$-dissipative solvers for scalar conservation laws with discontinuous flux. Arch. Ration. Mech. Anal., 201(1): 27-86, 2011.

[8] K. Brenner, C. Cancès, and D. Hilhorst. Convergence of finite volume approximation for immiscible two-phase flows in porous media with discontinuous capillary pressure field in several dimensions. HAL: hal-00675681, 2012 (submitted).

[9] R. Bürger, H. Frid and K.H. Karlsen. On the well-posedness of entropy solutions to conservation laws with a zero-flux boundary condition. J. Math. Anal. Appl. 326: 108-120, 2007.

[10] R. Bürger, K. H. Karlsen, and J. D. Towers. An Engquist-Osher-type scheme for conservation laws with discontinuous flux adapted to flux connections. SIAM J. Numer. Anal., 47(3): $1684-1712,2009$.

[11] F. Buzzi, M. Lenzinger, and B. Schweizer. Interface conditions for degenerate two-phase flow equations in one space dimension. Analysis, 29: 299-316, 2009.

[12] C. Cancès. Finite volume scheme for two-phase flow in heterogeneous porous media involving capillary pressure discontinuities. M2AN Math. Model. Numer. Anal., 43: 973-1001, 2009.

[13] C. Cancès. Asymptotic behavior of two-phase flows in heterogeneous porous media for capillarity depending only on space. I. Convergence to the optimal entropy solution. SIAM J. Math. Anal., 42(2): 946-971, 2010.

[14] C. Cancès. Asymptotic behavior of two-phase flows in heterogeneous porous media for capillarity depending only on space. II. Nonclassical shocks to model oil-trapping. SIAM J. Math. Anal., 42(2): 972-995, 2010.

[15] C. Cancès. On the effects of discontinuous capillarities for immiscible two-phase flows in porous media made of several rock-types. Netw. Heterog. Media, 5(3): 635-647, 2010.

[16] C. Cancès, Th. Gallouët, and A. Porretta. Two-phase flows involving capillary barriers in heterogeneous porous media. Interfaces Free Bound., 11(2): 239-258, 2009.

[17] C. Cancès and M. Pierre. An existence result for multidimensional immiscible two-phase flows with discontinuous capillary pressure field. SIAM J. Math. Anal. 44(2): 966-992, 2012.

[18] G. Chavent, G. Cohen and J. Jaffré. A Finite-Element simulator for incompressible two-phase flow. Transp. Porous Media 2: 465-478, 1987. 
[19] G. Chavent and J. Jaffré. Mathematical Models and Finite Elements for Reservoir Simulation: Single Phase, Multiphase, and Multicomponent Flows Through Porous Media. Elsevier, 1986

[20] S. Diehl. A uniqueness condition for nonlinear convection-diffusion equations with discontinuous coefficients. J. Hyperbolic Diff. Equ. 6(1): 127-159, 2009.

[21] G. Enchéry, R. Eymard, and A. Michel. Numerical approximation of a two-phase flow in a porous medium with discontinuous capillary forces. SIAM J. Numer. Anal., 43(6): 2402-2422, 2006.

[22] R. Eymard, T. Gallouët, and R. Herbin. Finite Volume Methods. Handbook of Numerical Analysis, Vol. VII, P. Ciarlet, J.-L. Lions, eds., North-Holland, 2000.

[23] R. Eymard, C. Guichard, R. Herbin and R. Masson. Gradient schemes for two-phase flow in heterogeneous porous media and Richards equation. HAL : hal-00740367, 2012 (submitted).

[24] E. F. Kaasschieter. Solving the Buckley-Leverett equation with gravity in a heterogeneous porous medium. Comput. Geosci., 3(1): 23-48, 1999.

[25] S. N. Kruzhkov. First order quasilinear equations with several independent variables. Mat. Sb. 81(123): 228-255, 1970. 